\title{
Quantification and Clustering of Inorganic Nanoparticles in Wastewater Treatment Plants across Switzerland"
}

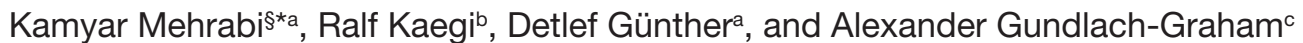

\$METAS Award 2020

\begin{abstract}
Single particle Inductively Coupled Plasma Time-of-Flight Mass Spectrometry (sp-ICP-TOFMS), in combination with online microdroplet calibration, allows the determination of particle number concentrations (PNCs) and the masses of elements in individual particles. Because sp-ICP-TOFMS analyses of environmental samples produce rich datasets composed of both single-metal nanoparticles (smNPs) and many types of multimetal NPs (mmNPs), interpretation of these data is well suited to automated analysis schemes. Here, we present a data analysis approach that includes automatic particle detection and elemental mass determinations based on online microdroplet calibration, and unsupervised clustering analysis of mmNPs to identify unique classes of NPs based on their element compositions. To demonstrate the potential of our approach, we analyzed wastewater samples collected from the influent and effluent of five wastewater treatment plants (WWTPs) across Switzerland. We determined elemental masses in individual NPs, as well as PNCs, to estimate the NP removal efficiencies of the individual WWTPs. Through hierarchical clustering, we identified NP classes conserved across all WWTPs; the most abundant particle types were those rich in $\mathrm{Ce}-\mathrm{La}, \mathrm{Fe}-\mathrm{Al}, \mathrm{Ti}-\mathrm{Zr}$, and $\mathrm{Zn}-\mathrm{Cu}$. In addition, we found particle types that are unique to one or a few WWTPs, which could indicate point sources of anthropogenic NPs.
\end{abstract}

Keywords: Clustering $\cdot$ ICP-TOFMS $\cdot$ Microdroplet calibration $\cdot$ Nanoparticle $\cdot$ Wastewater

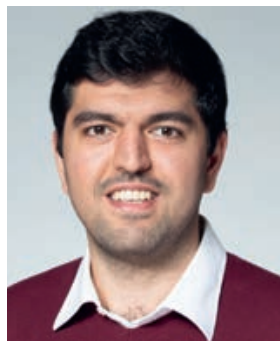

Kamyar Mehrabi obtained his Bachelor's degree from Sharif University of Technology, Tehran, Iran and his Master's degree from EPF Lausanne in material science and engineering. During his Master's studies he was a visiting researcher at the Swiss Federal Laboratories for Materials Science and Technology (Empa) in St. Gallen and Brigham and Women's Hospital (BWH) in Boston, USA. In 2018, he started his doctoral studies in the group of Prof. Detlef Günther at ETH Zürich on multiplexed nanoparticle analyses. He was awarded the METAS prize 2020 for his work on an online microdroplet calibration method to compensate for matrix effects in single-particle ICP-TOFMS.

\#Parts of this article have been published in open access form (CC-BY licence) K. Mehrabi, R. Kaegi, D. Günther, A. Gundlach-Graham, 'Emerging Investigator Series: Automated Single-Nanoparticle Quantification and Classification: A Holistic Study of Particles into and out of Wastewater Treatment Plants in Switzerland', Environ. Sci.: Nano 2021, 8, 1211-1225, https://doi.org/10.1039/d0en01066a. Reproduction is with permission from the Royal Society of Chemistry.

${ }^{*}$ Correspondence: K. Mehrabia, E-mail: kamyarm@inorg.chem.ethz.ch aLaboratory of Inorganic Chemistry, ETH Zurich, Switzerland;

${ }^{b}$ Department of Process Engineering, Eawag, Dübendorf, Switzerland;

'Department of Chemistry, lowa State University, Ames, USA

\section{Introduction}

In ICP-TOFMS, the complete elemental mass spectrum (from $\mathrm{Li}$ to $\mathrm{U}$ ) is recorded continuously at time resolutions down to $1 \mathrm{~ms}$. At this time resolution, ICP-TOFMS can be used to record NPderived signals, which are short transients typically between 300 $500 \mu$ s in duration. ${ }^{[1]}$ Single particle-ICP-TOFMS can be used to quantitatively determine the amounts of several elements and isotopes in single NPs. Through multi-element and multi-isotope detection, sp-ICP-TOFMS provides a more comprehensive analysis of the composition of individual NPs, which is ideal for clustering, sorting, and identifying unique NP types from particle mixtures that occur in environmental samples. However, some elements commonly present in NPs, such as carbon, nitrogen, oxygen, sulphur, and fluorine, are not readily detectable at the single-particle level by ICP-TOFMS. [2] Throughout this article, we use the terms 'single-metal' and 'multi-metal' NPs (smNP and $\mathrm{mmNP}$ ) to refer to particles measured with just one and with two or more ICP-TOFMS-detectable elements, respectively.

Distinguishing between naturally occurring NPs (NNPs) and engineered NPs (ENPs) has been the topic of several studies.[3] Although there is ambiguity on the best method of investigation of these particle types, the most prominent NP characteristics used to categorize a NP as either engineered or natural include composition, crystal structure, particle morphology, and shape. ${ }^{[4]}$ With sp-ICP-TOFMS, the simultaneous measurement of most metal and metalloid elements allows for the detection of variable and unique elemental fingerprints from different particle types. Due to manufacturing processes, it is assumed that ENPs will have fairly pure and controlled elemental fingerprints, which will differ from those of NNPs. ${ }^{[5]}$ However, human-based evaluation of elemental associations through visual inspection of data and user-based pattern recognition is extremely time consuming and 
is prone to user error and bias. ${ }^{[6]}$ For example, a user might limit the analyte element list to simplify multi-metal patterns in the data and so miss unanticipated multi-metal associations. Similarly, low-abundance multi-metal associations are more likely to be overlooked in non-automated data analysis schemes.

In this article, we show a high-throughput data evaluation approach for non-targeted analysis of NPs measured via sp-ICPTOFMS. We apply these techniques to extract NP information from sp-ICP-TOFMS measurements of influent and effluent from five wastewater treatment plants (WWTPs) across Switzerland. Through the analysis of mmNP compositions, we report a means - via hierarchical clustering - to discover both conserved and unique $\mathrm{mmNP}$ types across the wastewater samples. This $\mathrm{mmNP}$ clustering approach provides insights into the origins of various nanoparticle types present in the wastewater samples.

\section{Experimental Approach}

\subsection{Sample Selection and Preparation}

We collected influent (I) samples after primary clarification and effluent (E) samples in $500 \mathrm{~mL}$ glass media bottles from five WWTPs (Fig. 1) and measured the samples by sp-ICP-TOFMS on the day of collection. Prior to sp-ICP-TOFMS analysis, we sonicated the samples. More details on sample preparation can be found in the original article. ${ }^{[7]}$

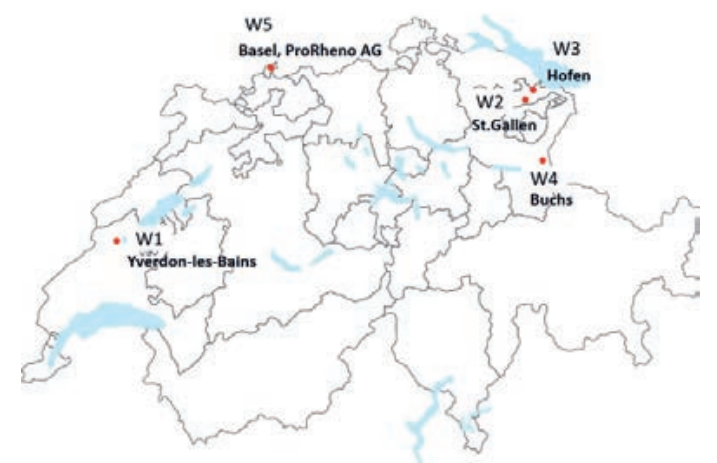

Fig. 1 WWTP sampling sites. ${ }^{[8]}$ For each site, the respective locality as well as the abbreviation for it in the main text are highlighted. Influent samples are indicated by ' $\mathrm{l}$ ' and effluent by ' $\mathrm{E}$ '. This figure is reproduced from Environ. Sci.: Nano, 2021, 8, 1211-1225 with permission from the Royal Society of Chemistry.

\subsection{Sample Introduction and MS Measurement}

Measurements were performed on an icpTOF-2R (TOFWERK AG, Thun, Switzerland) instrument, which has a native mass resolving power $(\mathrm{m} / \Delta m$ at $\mathrm{FWHM})$ of $\sim 6000$ and an extraction frequency of $21.739 \mathrm{kHz}$. Element-mass and PNC determinations were performed according to our previously published online microdroplet calibration method. ${ }^{[9]}$ As shown in Fig. 2, we used a dual-sample introduction setup, which was composed of nebulizer/spray chamber for wastewater sample introduction and a falling tube and microdroplet generator $(50-\mu \mathrm{m}$ diameter Autodrop Pipette, AD-KH-501-L6, Microdrop Technologies $\mathrm{GmbH}$, Germany) for introduction of microdroplets calibration standards. Droplets were introduced into a vertical falling tube filled with a helium/argon gas ${ }^{[10]}$ mixture and then introduced into the plasma concomitant with sample aerosols from the conventional pneumatic nebulizer/cyclonic spray chamber. A known amount of a plasma-uptake standard (i.e. cesium, Cs) was spiked into the WWTP samples and the microdroplet calibration solution. Through detection of the nebulized and microdropletcontained $\mathrm{Cs}$, we determined a sample plasma uptake rate for every sample, which allowed us to directly calculate PNCs.

\subsection{Data Processing}

We developed an automated data analysis procedure to detect, quantify, and cluster smNPs and mmNPs for sp-ICP-TOFMS data. With online microdroplet calibration, we determined the mass of detectable elements in individual particles and the PNCs for each type of found NP. ${ }^{[7,9,11]}$ In addition, our sp-ICP-TOFMS particles detection and clustering software, 'TOF_NanoFinder' is available under an open-source license (http://doi.org/10.5905/ ethz-1007-363) and is hosted at https://github.com/ethz-tema/ TEMAsingleParticle.

We only clustered the multi-metal particles (mmNPs). For smNPs, we obtain mass distributions and PNCs; however, this information is not sufficient to develop meaningful clusters of the smNPs. Therefore, smNPs can be thought of as each representing their own particle cluster. If we had additional information about smNPs, such as shape or crystal structure, we could consider developing clustering approaches to identify the most similar smNP families.

\section{Automated Clustering to Discover Multi-metal Nanoparticle Types}

The possibility to differentiate between natural and anthropogenic NPs based on multi-metal fingerprints has received considerable interest. ${ }^{[12]}$ However, as an inventory of natural and
Fig. 2. Schematic diagram of sp-ICP-TOFMS with online microdroplet calibration. The sample is delivered to the ICP as a fine aerosol via the pneumatic nebulizer. Calibration microdroplets are dried in a falling tube before joining the nebulized sample through T-piece, and injected into the ICP. Inserted graph: time trace of elements monitored in a sample. Two droplet burst signals are visible at both ends of the time trace. The horizontal green line represents the dissolved uptake standard signal, and vertical orange lines represent analyte signals from microdroplets and are nanoparticles.

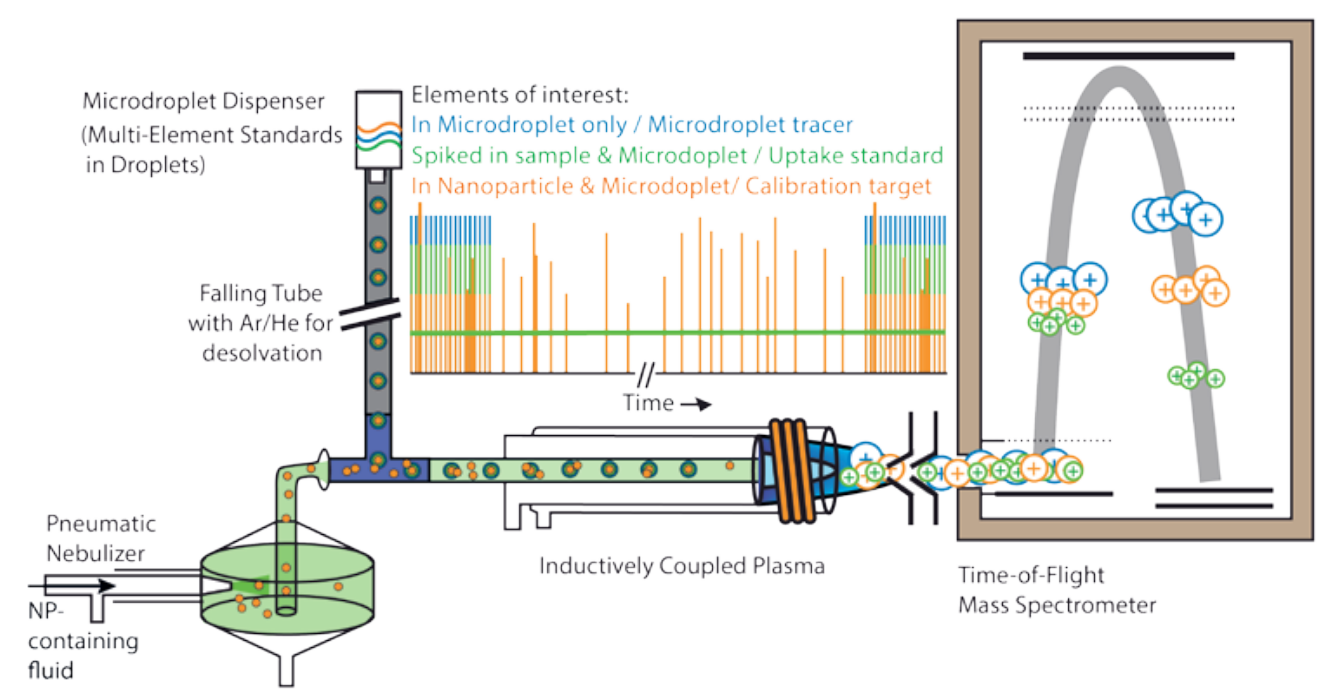


anthropogenic particle types is currently lacking, NP identification based on database searching or spectral matching approaches is not possible. To identify different clusters of mmNPs - which may be assigned to specific sources - we developed a two-stage hierarchical clustering analyses. Clustering was accomplished with a custom-written MATLAB (verR2020b Mathworks, MA, USA) script, accessible by TOF_NanoFinder software; a schematic of our clustering approach is provided in Fig. 3. In the first step of $\mathrm{HC}$, we performed intra-sample clustering of elemental masses present in each mmNP using the correlation distance and averaging method for HC. After calculating the average correlation distance between all the particle-events, we generated an agglomerative hierarchical cluster tree, in which each particle is connected to the cluster tree by a single linkage and linkages are merged together to minimize average distance between all mmNPs. We used an empirically determined cut-off distance of 0.85 to identify major clusters in each wastewater sample, and then created representative mmNP proxies for each of the major clusters found. The mmNP proxies were composed of elements that occurred in more than $10 \%$ of the particles part of given cluster and the amplitude of elements in mmNP proxies was the median of the ratio of element mass to the mass of the most-frequent occurring element in the cluster.

In order to compare the similarities between the mmNP clusters developed for the different WWTPs, we performed a second inter-sample $\mathrm{HC}$ analysis. In this analysis, we clustered a pooled sample of the representative mmNP proxies from the first HC. For inter-sample clustering of the mmNP proxies, we again used the correlation distance metric with average unweighted linkages, but used a lower cluster cut-off distance of 0.5 to define the major clusters. Subgroups of the major intra-sample clusters, which break up the major clusters into contributions from different WWTPs, are apparent below correlation distances of 0.5 on the resultant dendrogram (see Fig. 5).

\section{WWTPs in Terms of Nanoparticle Removal}

In Fig. 4, we present PNCs for several smNPs and clusters of mmNPs measured in the influent and effluent of the five WWTPs. While we find many of the same particle clusters in the influent and effluent, there are dramatically fewer NPs in the effluent. This detailed view of removal efficiency by particle type demonstrates that, in our analyses, NPs composed primarily of $\mathrm{Al}, \mathrm{Ti}, \mathrm{Fe}, \mathrm{Cu}$, $\mathrm{Zn}$, and $\mathrm{Ce}$ are the dominant particle types in both influent and effluent, but are efficiently removed in most cases. (It is likely that silicate $\left(\mathrm{SiO}_{2}\right) \mathrm{NPs}$, which we could not measure at the singleparticle level due to low sensitivity at mass-to-charge $(\mathrm{m} / \mathrm{z}) 28$ with the instrument settings used, would also be a dominant NNP species.) Interestingly, some particle types seem to be less retained in WWTPs than others. For example, there is a larger fraction of Fe-containing smNPs in the effluent than other particle types. The apparent low removal efficiency of Fe NPs in terms of particle number could be due to the addition of iron in the wastewater treatment process to retain phosphorus; ${ }^{[13]}$ some of the created 'Fe-P' colloids may pass the wastewater treatment. Through the characterization of mmNPs, we also find that most NP types are removed efficiently from the wastewater stream. Overall, the most abundant mmNP types include Ti-Zr, $\mathrm{Zn}-\mathrm{Cu}$, $\mathrm{Al}-\mathrm{Fe}$, and Ce-La. Conservation of NP type and abundance across all WWTPs suggests that the NPs are naturally occurring and ubiquitous. On the other hand, we find some NP types such as of Rh-Pd or Bi-V in just one or two WWTPs, which suggests that these NP types originate from specific (anthropogenic) point sources. Furthermore, we found substantial differences in the numbers of mmNPs detected in the influent and effluent samples from the different WWTPs. With these high mmNP fractions, significant information content on the description of NPs populations entering and exiting WWTPs is associated with the composition of mmNPs.

\section{Characterization of Multi-metal NP Clusters}

In Fig. 5, we present results from two-stage $\mathrm{HC}$ analysis of the mmNP data from the influent of WWTPs. The major clusters from each WWTP are 'leaves' in the cluster tree; names of each leaf correspond to the wastewater samples (i.e. I1-I5) and the most frequent elements in the mmNP cluster. Branches of the cluster tree show the relation of mmNP clusters across the different WWTPs. Overall, we identify $23 \mathrm{mmNP}$ clusters across the five WWTP samples. The dendrogram reveals that some mmNP clusters are conserved across all WWTPs and other branches result from one or a few WWTPs. While HC analysis reduces mmNP data into 23 clusters and 77 leaves; the original data from each of these mmNPs is still accessible. Cluster tree representation offers the possibility to select branches or leaves of interest, and then extract and examine mmNP data from that subset. For example, one can evaluate the PNCs or element masses per particle for any given branch or leaf. For the remainder of this manuscript, we explore selected aspects of the mmNP dataset that become apparent through clustering analysis.

The dendrogram in Fig. 5a shows that some clusters of mmNPs are more conserved than others: mmNP types that exist in all samples include those rich in Ti-Zr, $\mathrm{Zn}-\mathrm{Cu}, \mathrm{Au}-\mathrm{Ag}, \mathrm{Ce}-\mathrm{La}$, and $\mathrm{Zr}-\mathrm{Y}$. The ubiquity of these mmNP types suggests a natural
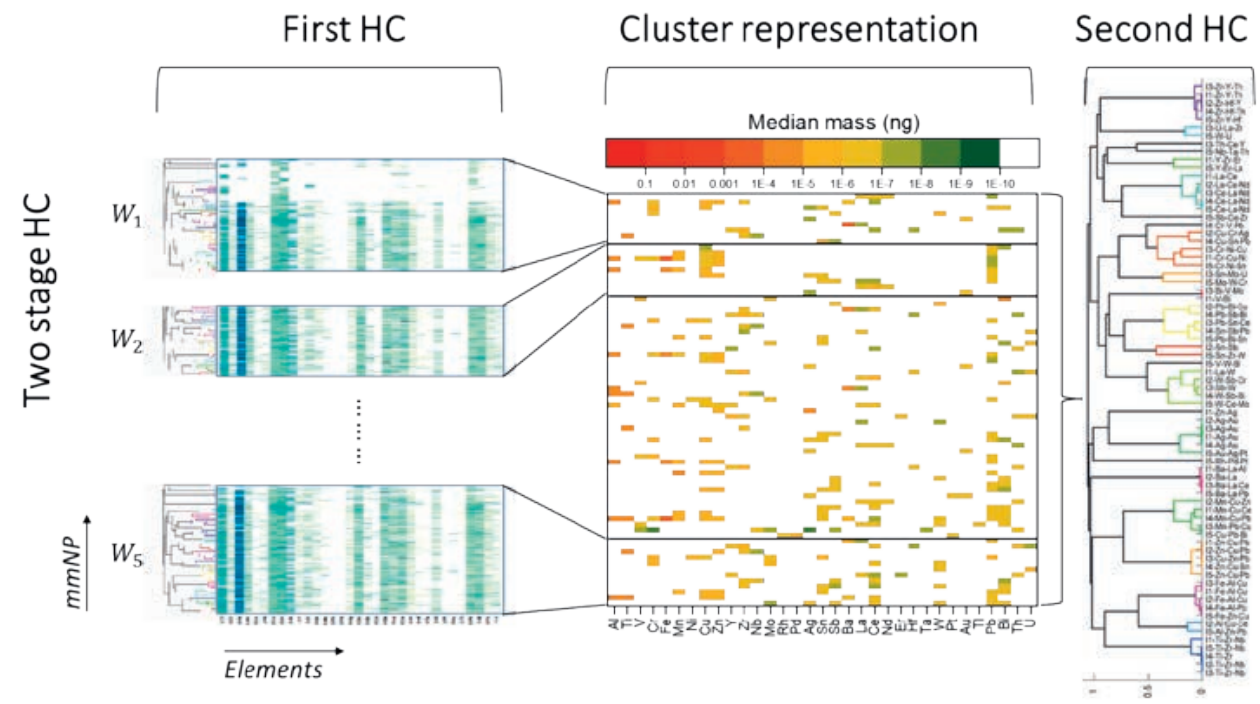

Fig. 3. Graphical illustration of two-stage hierarchical clustering $(\mathrm{HC})$ of mmNPs. In the first step, $\mathrm{HC}$ is performed on individual samples. Then, a representative for each cluster from each sample is determined as described in the manuscript. These representative mmNP proxies are clustered again to find inter-sample clusters and unique mmNP types. T This figure is reproduced from Environ. Sci.: Nano 2021, 8, 1211-1225 with permission from the Royal Society of Chemistry. 

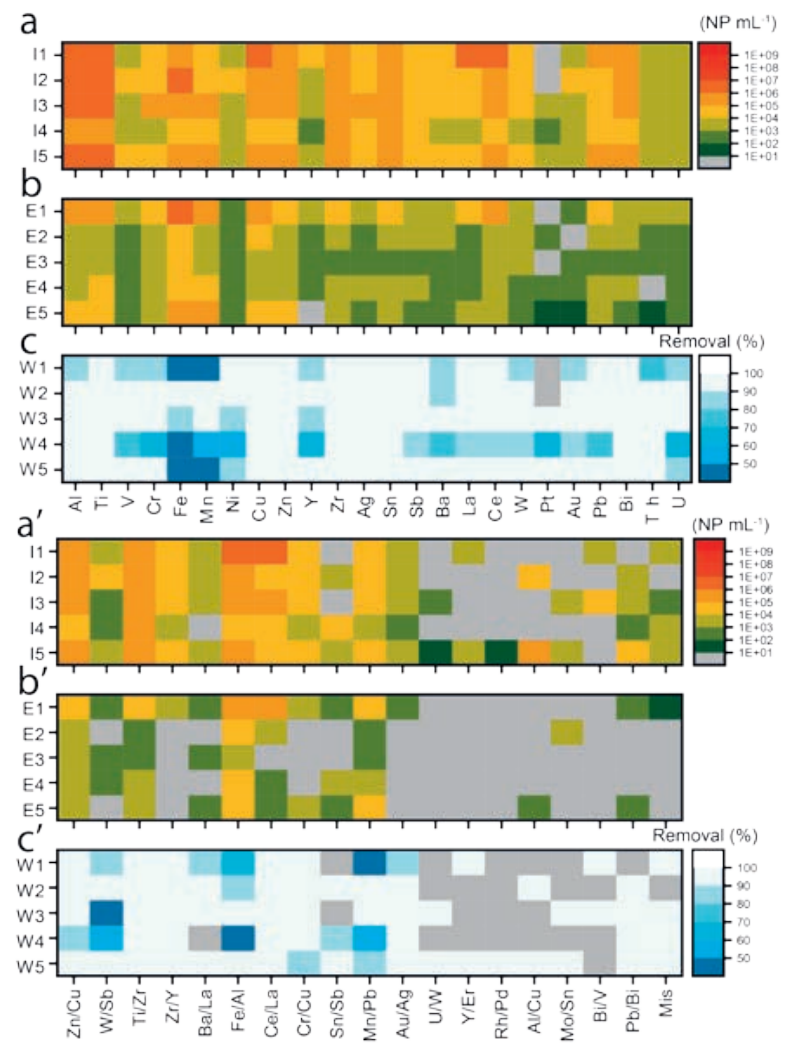

Fig. 4. smNPs and mmNPs detection across different WWTPs. Heat maps show the PNCs in the influent (a) and effluent (b), and the PNCbased removal efficiency of each particle type in percentage (c). mmNP clusters are labelled according to the two most abundant elements measured in each particle cluster. Classification of the mmNPs is obtained via hierarchical clustering analysis as described in text (cf. Figs. 3, 5). This figure is reproduced from Environ. Sci.: Nano 2021, 8 , 1211-1225 with permission from the Royal Society of Chemistry. origin, or at least common anthropogenic sources: if mmNPs were from specific point sources of anthropogenic NP pollution, they would likely be found only in selected WWTPs. In addition, we know from previous research that our mmNP types match, at least in part, results from studies focused on $\mathrm{Ce}, \mathrm{Ti}, \mathrm{Zr}$, and $\mathrm{Zn}$ natural NPs. ${ }^{[3,5,12,14]}$ In Fig. 5b-g, we extract data from selected mmNP clusters and plot the normalized mass of elements that are measured in at least $1 \%$ of the particles that make up each clusters. Normalization was done at the particle level: the quantified masses of each element were divided by the mass of the most frequently occurring element from the cluster. For example, in Fig. 3b-g, the normalizing elements were $\mathrm{Ti}, \mathrm{Zn}, \mathrm{Ag}, \mathrm{V}, \mathrm{Ce}$, and $\mathrm{Zr}$, respectively. The rather well conserved element ratios in these mmNP clusters further emphasizes that these mmNP clusters likely originate from comparable sources and are likely naturally occurring.

In addition to ubiquitous NNPs, hierarchical clustering also identifies uncommon particle types including NPs rich in AuAg, Rh-Pd, Bi-V-(Mo), Sb-W, Cr-Ni, Zn-Cu, Mn-Cu, and Ba-La. We find a Bi-V-rich mmNP cluster in two of the WWTP influent samples. As shown in Fig. 5e, the mean mass ratios of $\mathrm{Bi}: \mathrm{V}$ in this cluster are 5:1 in I3 and 6.8:1 in I1. Because ${ }^{209} \mathrm{Bi}$ is about four-times heavier than ${ }^{51} \mathrm{~V}$, the measured element ratios are reasonably consistent with a one-to-one atomic ratio $(4.1: 1, \mathrm{Bi}: \mathrm{V}$ mass ratio) of bismuth vanadate $\left(\mathrm{BiVO}_{4}\right)$, especially considering the low numbers of particles recorded. Bismuth vanadate - which is sometimes doped with Mo (as also found in the Bi-V-Mo cluster from I3) - is used as yellow pigment in industry ${ }^{[15]}$ and as a catalyst, ${ }^{[16]}$ and is very likely anthropogenic in origin. Another unexpected class is the Au-Ag cluster (Fig. 3d) that contains 5-10 times more $\mathrm{Au}$ than $\mathrm{Ag}$ on a mass basis. We find the $\mathrm{Au}-\mathrm{Ag}$ cluster in all WWTP samples from both of our collection days. These NPs may originate from gold alloys used in the jewellery manufacturing industry, and thus are likely anthropogenic in origin.
Fig. 5. a). Dendrogram of hierarchical clustering analysis of samples 11-15. In this cluster tree, we identify 23 major mmNP types. Each unique mmNP type is plotted in a different color. Six of the unique mmNP clusters are highlighted in colored rectangles. For these mmNP clusters, the representative mmNP proxies with elements present at an occurrence rate limit of $1 \%$ are plotted in b-g. Note that not all mmNPs types are found in all WWTP influent samples. While the Ti-Zr, $\mathrm{Zn}-\mathrm{Cu}, \mathrm{Au}-\mathrm{Ag}, \mathrm{Ce}-\mathrm{La}$, and $\mathrm{Zr}-\mathrm{Y}$ $\mathrm{mmNP}$ clusters are found in all influent samples, the Bi-V-type mmNPs are only found in $\mathrm{I} 1$ and 13. Error bars mean in b-g are standard deviation of normalized masses. This figure is reproduced from Environ. Sci.: Nano 2021, 8, 1211-1225 with permission from the Royal Society of Chemistry.
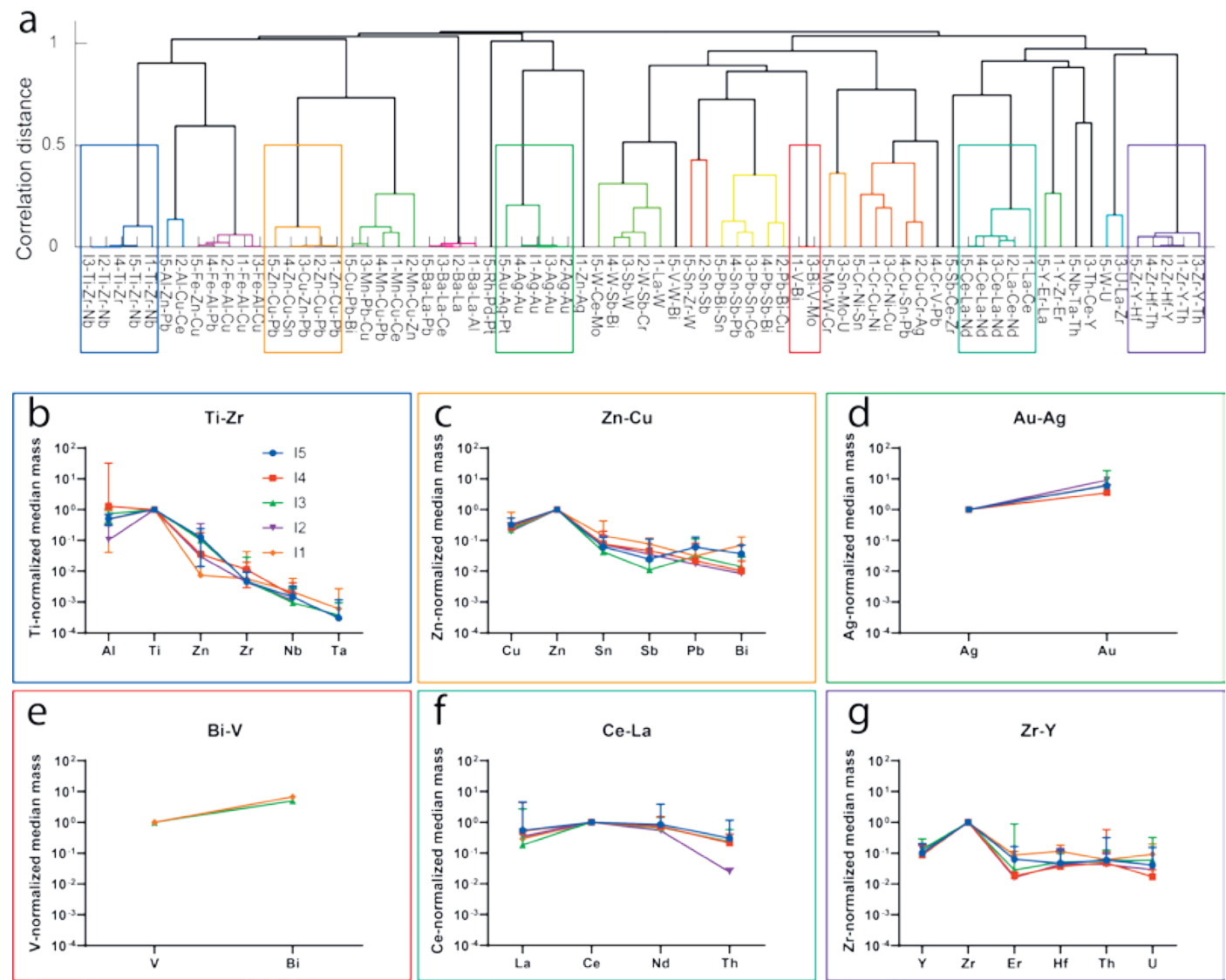


\section{Conclusions}

We presented a strategy for the analysis of NPs in environmental waters or other water samples. Wastewater samples are complex and likely have a mix of different types of natural and anthropogenic NPs. Here, we created rich sp-ICP-TOFMS datasets composed of a range of smNPs and mmNP types. To break down the inherent complexity of sp-ICP-TOFMS datasets and reduce them to an interpretable form, we combined online microdroplet calibration with an automated data analysis system. With this approach, we are able to extract NP data from raw ICP-TOFMS time traces, determine the element masses in single NPs, record PNCs, correct the data sets for the presence of particle-coincidence events, and cluster mmNPs in an unsupervised fashion. Until now, a major bottleneck in sp-ICP-TOFMS analysis was data processing. However, we are now able to rapidly deliver multi-element quantitative analysis of NP populations in real samples, and to provide initial clustering analysis to aid the interpretation of the data - for example, through the identification of unique mmNP types.

Interpretation of our sp-ICP-TOFMS data set in terms of NP origin is an ongoing process and we expect continued refinement of our understandings. In fact, the continued refinement of our knowledge about mmNP fingerprints (both of natural and anthropogenic NP types) and the expected types of mmNPs in various environmental compartments is a substantial benefit of our current measurement approach. Because our approach allows for both high-throughput data collection and data analysis, we are now able to expand our inventory of NPs - which will, in turn, improve our classification models. An eventual aim of such studies is to establish a database approach for the identification of different NP types.

\section{Acknowledgements}

The authors wish to thank Matthias Philipp from Eawag (Dübendorf, Switzerland) for collection of the WWTPs samples, and also the operators of the WWTPs for access to samples. The authors thank Roland Mäder from the ETH mechanical workshop for manufacturing components of the dual sample introduction setup. A. G.-G. and K.M.K. acknowledge funding through an Ambizione grant of the SNSF, project no. PZ00P2_174061.

Received: June 23, 2021

[1] O. Borovinskaya, S. Gschwind, B. Hattendorf, M. Tanner, D. Gunther, Anal. Chem. 2014, 86, 8142, https://doi.org/10.1021/ac501150c.

[2] I. Kálomista, A. Kéri, G. Galbács, J. Anal. At. Spectrom. 2016, 31, 1112, https://doi.org/10.1039/C5JA00501A.

[3] M. M. Nabi, J. Wang, M. Baalousha, Chemosphere 2021, 263, 128261, https://doi.org/10.1016/j.chemosphere.2020.128261.

[4] F. von der Kammer, P. L. Ferguson, P. A. Holden, A. Masion, K. R. Rogers, S. J. Klaine, A. A. Koelmans, N.

Horne, J. M. Unrine, Environ. Toxicol. Chem. 2012, 31, 32, https://doi.org/10.1002/etc.723.

[5] A. Praetorius, A. Gundlach-Graham, E. Goldberg, W. Fabienke, J. Navratilova, A. Gondikas, R. Kaegi, D. Gunther, T. Hofmann, F. von der Kammer, Environ. Sci.: Nano 2017, 4, 307, https://doi.org/10.1039/c6en00455e.

[6] A. Gondikas, F. von der Kammer, R. Kaegi, O. Borovinskaya, E. Neubauer, J. Navratilova, A. Praetorius, G. Cornelis, T. Hofmann, Environ. Sci.: Nano 2018, 5, 313, https://doi.org/10.1039/c7en00952f.

[7] K. Mehrabi, R. Kaegi, D. Gunther, A. GundlachGraham, Environ. Sci.: Nano 2021, $8, \quad 1211$, https://doi.org/10.1039/d0en01066a.

[8] A. Gogos, J. Wielinski, A. Voegelin, F. V. Kammer, R. Kaegi, Water. Res. X 2020, 9, 100059, https://doi.org/10.1016/j.wroa.2020.100059.

[9] K. Mehrabi, D. Gunther, A. Gundlach-Graham, Environ. Sci.: Nano 2019, 6, 3349, https://doi.org/10.1039/c9en00620f.

[10] B. Ramkorun-Schmidt, S. A. Pergantis, D. Esteban-Fernandez, N. Jakubowski, D. Gunther, Anal. Chem. 2015, 87, 8687, https://doi.org/10.1021/acs.analchem.5b01604.

[11] a) A. Gundlach-Graham, L. Hendriks, K. Mehrabi, D. Gunther, Anal. Chem. 2018, 90, 11847, https://doi.org/10.1021/acs.analchem.8b01551; b) L. Hendriks, A. Gundlach-Graham, D. Gunther, Chimia 2018, 72, 221, https://doi.org/10.2533/chimia.2018.221; c) L. Hendriks, B. RamkorunSchmidt, A. Gundlach-Graham, J. Koch, R. N. Grass, N. Jakubowski, D. Gunther, J. Anal. At. Spectrom. 2019, 34, 716, https://doi.org/10.1039/ c8ja00397a; d) L. Hendriks, A. Gundlach-Graham, D. Gunther, J. Anal. At. Spectrom. 2019, 34, 1900, https://doi.org/10.1039/c9ja00186g; e) A Gundlach-Graham, K. Mehrabi, J. Anal. At. Spectrom. 2020, 35, 1727, https://doi.org/10.1039/d0ja00213e.

[12] a) J. Wang, M. M. Nabi, S. K. Mohanty, A. N. Afrooz, E. Cantando, N. Aich, M. Baalousha, Chemosphere 2020, 248, 126070, https:// doi.org/10.1016/j.chemosphere.2020.126070; b) M. Baalousha, J. Wang, M. M. Nabi, F. Loosli, R. Valenca, S. K. Mohanty, N. Afrooz, E. Cantando, N. Aich, J. Hazard. Mater. 2020, 392, 122335, https://doi.org/10.1016/j.jhazmat.2020.122335.

[13] P. Wilfert, P. S. Kumar, L. Korving, G. J. Witkamp, M. C. van Loosdrecht, Environ. Sci. Technol. 2015, 49, 9400, https://doi.org/10.1021/acs.est.5b00150.

[14] A. Azimzada, J. M. Farner, I. Jreije, M. Hadioui, C. Liu-Kang, N. Tufenkji, P. Shaw, K. J. Wilkinson, Front. Environ. Sci. Eng. 2020, 8, 91, https://doi.org/10.3389/fenvs.2020.00091.

[15] S. Schwarz, H. Endriss, Rev. Prog. Color. Relat. Top. 1995, 25, 6.

[16] K. Schuh, W. Kleist, M. Hoj, V. Trouillet, A. D. Jensen, J. D. Grunwaldt, Chem. Commun. 2014, 50, 15404 , https://doi.org/10.1039/c4cc07527g.

\section{License and Terms}

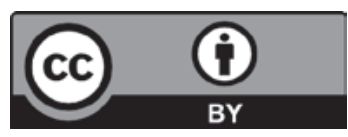

This is an Open Access article under the terms of the Creative Commons Attribution License CC BY 4.0. The material may not be used for commercial purposes.

The license is subject to the CHIMIA terms and conditions: (http:// chimia.ch/component/sppagebuilder/?view=page \&id=12).

The definitive version of this article is the electronic one that can be found at https://doi.org/10.2533/chimia.2021.642 